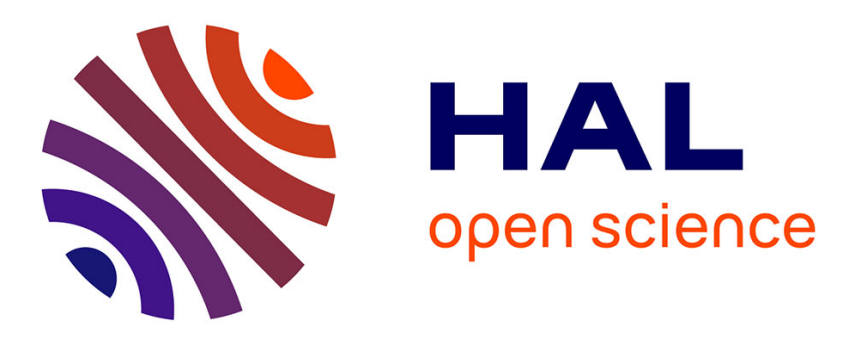

\title{
Foamability and stability of foams obtained with silica/PEI gels
}

Rémi Deleurence, Clément Freymond, Tamar Saison, François Lequeux, Cécile Monteux

\section{- To cite this version:}

Rémi Deleurence, Clément Freymond, Tamar Saison, François Lequeux, Cécile Monteux. Foamability and stability of foams obtained with silica/PEI gels. Colloids and Surfaces A: Physicochemical and Engineering Aspects, 2017, 534, pp. 2-7. 10.1016/j.colsurfa.2017.05.093 . hal-01548166

\section{HAL Id: hal-01548166 https: / hal.sorbonne-universite.fr/hal-01548166}

Submitted on 27 Jun 2017

HAL is a multi-disciplinary open access archive for the deposit and dissemination of scientific research documents, whether they are published or not. The documents may come from teaching and research institutions in France or abroad, or from public or private research centers.
L'archive ouverte pluridisciplinaire HAL, est destinée au dépôt et à la diffusion de documents scientifiques de niveau recherche, publiés ou non, émanant des établissements d'enseignement et de recherche français ou étrangers, des laboratoires publics ou privés. 


\title{
Foamability and stability of foams obtained
}

\section{with silica/PEI gels}

Rémi Deleurence ${ }^{\mathrm{a}, \mathrm{b}}$, Clément Freymond ${ }^{\mathrm{a}, \mathrm{b}}$, Tamar Saison ${ }^{\mathrm{c}}$, François Lequeux ${ }^{\mathrm{a}, \mathrm{b}}$ and Cécile

Monteux*a,b,d

\begin{abstract}
a Laboratoire Sciences et Ingénierie de la Matière Molle, CNRS, ESPCI Paris, PSL Research University, 10 rue Vauquelin, Paris, France

b Laboratoire Sciences et Ingénierie de la Matière Molle, Université Pierre et Marie Curie,

Sorbonne-Universités, 10 rue Vauquelin, France

c Saint-Gobain Recherche, 39 Quai Lucien Lefranc, 93300 Aubervilliers, France

d Global Station for Soft Matter, Global Institution for Collaborative Research and Education, Hokkaido University,

Sapporo, Hokkaido 060-0808, Japan

HighlightsPEI/silica mixtures, which are yield stress fluids, can be used to produce highly stable

foams.
\end{abstract}

There is an optimum silica concentration for which we obtain the highest foamability and the highest foam stability.

Graphical abstract

The amount of air incorporated during foaming decreases with particle concentration

The drainage rate decreases with the particle concentration
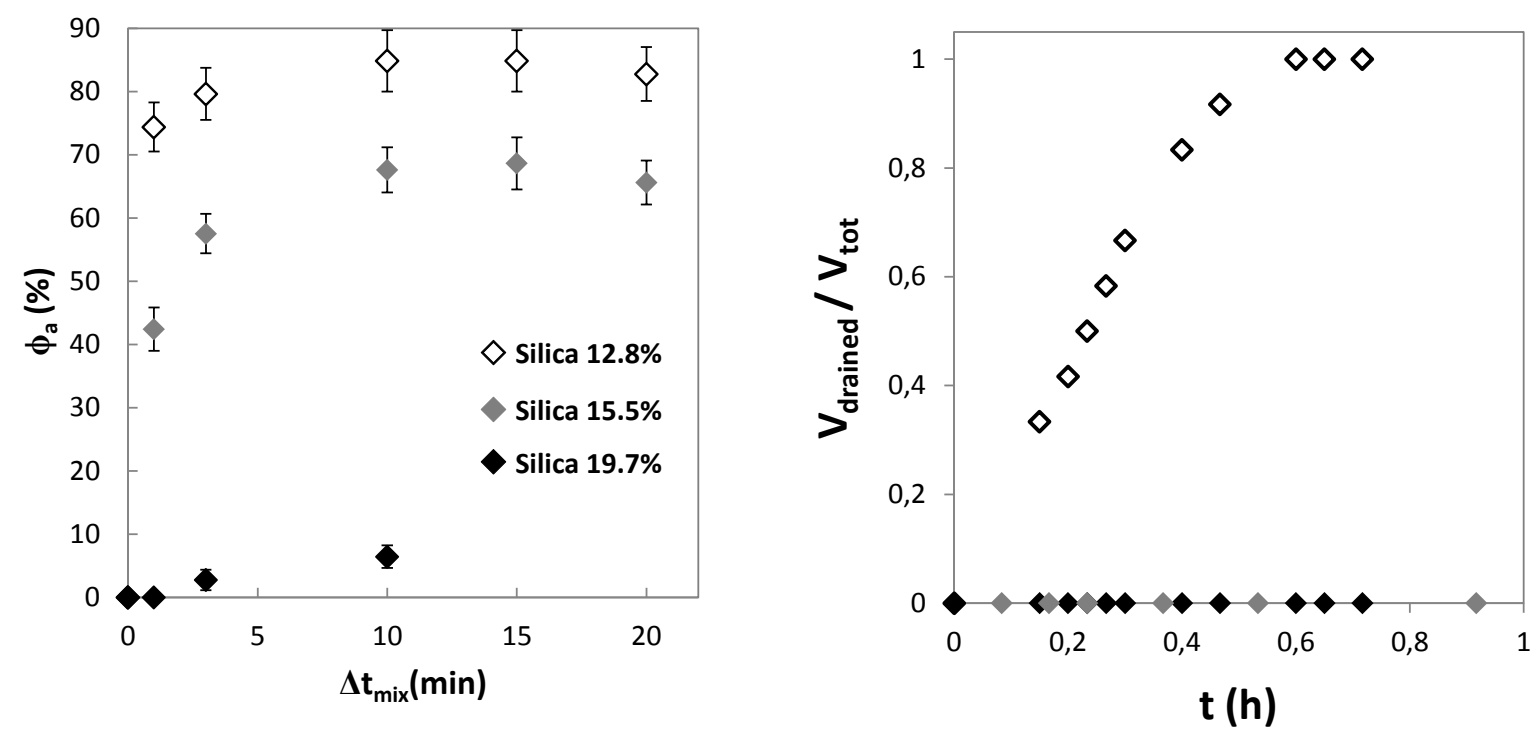

$<$ Inlinelmage1 $>$

\section{Abstract}

In this article we examine the foamability and foam stability of a series of PEI/silica gel suspensions with varying particle concentrations in the presence of a neutral surfactant, $\mathrm{C}_{12} \mathrm{E}_{23}$. These suspensions, 
which were recently developed for 3D printing applications, exhibit a finite yield stress whose value can be tuned by varying the particle concentration and the $\mathrm{pH}$. Our study aims at evaluating whether this system is suitable to produce stable foams that could be further be used as precursors for the $3 \mathrm{D}$ printing of porous materials and to evaluate the relation between the yield stress and the foam stability against drainage and ripening. At $\mathrm{pH} 7$ where all the suspensions are in the fluid state, the maximum air fraction reaches $80 \%$ however the foams are very unstable to drainage. At $\mathrm{pH} 10.5$, the suspensions are in the gel state due to Van der Waals driven aggregation of the particles. In the gel state, the obtained maximum air fraction decreases with the suspension concentration, from $\phi_{\text {air }}=80 \%$ for the $12.8 \mathrm{wt} \%$ suspension to $\phi_{\text {air }}=65 \%$ for the $15.5 \mathrm{wt} \%$ suspension. The most concentrated suspension, i.e $19.7 \mathrm{wt} \%$ could not be foamed. At pH 10.5, the yield stress of the $12.8 \mathrm{wt} \%$ suspension is $10 \mathrm{~Pa}$, which enables to overcome the buoyancy acting on the particles and therefore arrest drainage. However the yield stress is too low to overcome the Laplace pressure in the bubbles, therefore Ostwald ripening is observed. The $15.5 \mathrm{wt}$ $\%$ suspension exhibits a yield stress of $200 \mathrm{~Pa}$, which is high enough to arrest drainage. Ostwald ripening occurs at early times and is arrested when the bubble radius reaches $400 \mu \mathrm{m}$ as the yield stress equals the Laplace pressure.

The results obtained in this study show that there is an optimal silica concentration which combines good foamability and large stability to drainage and Ostwald ripening. These materials are promising for the development of 3D printable foams even though the size of the bubbles will limit the spatial resolution of the printing to a few millimeters.

Keywords: foams; particles; polymers

\section{Introduction}

Aqueous foams are composed of air bubbles dispersed in an aqueous phase. While the foaming process and stability of aqueous foams in which the continuous phase is newtonian have been extensively studied $^{1,2}$, the case of foams in which the continuous phase is a complex fluid has attracted less attention. Nevertheless, dispersing bubbles in complex fluids such as colloidal suspensions or polymer systems has numerous applications. For example, in the field of building materials, incorporating air into cement ${ }^{3}$ 
or plaster pastes enables the production of insulating and low density materials. Aeration of food products, ${ }^{4}$ which may enhance shelf life and create new textures, also requires to foam complex fluids. Recent studies have focused on foams made with complex fluids such as concentrated emulsions ${ }^{5,6}$, polymer gels ${ }^{7-10}$ or particle suspensions such as clays ${ }^{11-14}$, granular suspensions ${ }^{15,16}$, surfactant/particle suspensions ${ }^{12,13,17-22}$. When particles or particle aggregates are large enough, they accumulate in the Plateau borders of the foams and help slowing down the drainage of the foams ${ }^{15,16,23}$. When the particle suspensions exhibit a finite yield stress, this may lead to a complete arrest of the drainage flow ${ }^{17,24,25}$. Lesov et al. showed that their CAPB/silica system builds up a percolated network over time and that foam drainage is arrested when the yield stress of the network overcomes the hydrostatic pressure ${ }^{17}$. The same authors found that the Ostwald ripening can also be arrested, provided the yield stress is higher than the capillary pressure of the bubbles. Kloek et al. found numerically that ripening may be arrested for sufficiently high bulk elastic moduli of the continuous phase ${ }^{4}$.

Yield stress fluids exhibit an elastic response below a critical yield stress $\sigma_{0}$ and flow above $\sigma_{0}$. Among a large number of applications, yield stress fluids are promising systems for the development of new types of matrices that can be processed in 3D printing. Indeed 3D printing applications requires fluids that flow through a needle under a given stress and keep their shape at rest once deposited on a surface. In principle the same type of properties is required for producing stable foams. Indeed the foamability is enhanced as the viscosity decreases ${ }^{17}$ while a large elastic modulus as well as a yield stress enable arresting drainage and coarsening ${ }^{4,17}$.

In this article, we examine the foamability and foam stability of yield stress fluids obtained from suspensions containing PEI, Poly(ethylenimine), silica particles and a non ionic surfactant, $\mathrm{C}_{12} \mathrm{E}_{23}$. $\mathrm{PEI} /$ silica suspensions were recently developed for 3D printing applications ${ }^{26}$. It was shown that at $\mathrm{pH} 7$, the PEI chains contain protonated amine groups which are positively charged. The positively charged PEI chains adsorb on the negatively charged silica particles through strong electrostatic interactions. The resulting PEI/silica particles are positively charged and the corresponding suspensions exhibit a low shear modulus. As the $\mathrm{pH}$ is raised, the number of protonated groups on the PEI chains decreases and the neutralized PEI chains collapse on the silica particles and PEI/silica particles aggregate through Van 
der Waals forces. The corresponding mixtures exhibit high elastic moduli and yield stresses ranging from 10 to $10^{5} \mathrm{~Pa}$ depending on the particle concentration. In the present article, we study foams containing PEI/silica mixtures and $\mathrm{C}_{12} \mathrm{E}_{23}$. We study the foaming behavior of these mixtures as well as the stability of the obtained foams.

The two goals of our study are i) to evaluate whether this system is suitable to produce stable foams that could be further used as precursors for the 3D printing of porous materials. In this article we focus on the foamability and stability of the aqueous precursor foams. We focus on the influence of the particle concentration, which controls the yield stress and elastic modulus of the suspensions. ii) to evaluate the relation between the yield stress and the foam stability against drainage and ripening and compare it to previously published results.

We find that the maximum air fraction in the foams decreases with the particle concentration. However the stability of the foams increases with the yield stress of the suspensions. When the yield stress exceeds $10 \mathrm{~Pa}$, the drainage is completely arrested. We find that the Ostwald ripening cannot be arrested at early times as the capillary pressure in the bubbles exceeds the value of the yield stress. However at longer times, as the size of the bubbles increases, the capillary pressure decreases and eventually becomes equal to the yield stress. As a consequence, the size of the bubbles reaches a plateau around $400 \mu \mathrm{m}$. Our results show that there exists an optimum particle concentration which enables optimizing the amount of foam produced and its stability and that PEI/silica suspensions can be used as precursors for the production of porous materials.

\section{Materials and methods}

\subsection{Materials}

The PEI $\mathrm{M}_{\mathrm{w}}=2000 \mathrm{~g} / \mathrm{mol}$ was purchased from Sigma-Aldrich. The silica particles (Tixosil 365) were purchased from Solvay, the size of the particles ranges between 3.5 and $4 \mu \mathrm{m}$ and their specific area is $0.6 \mathrm{mg} / \mathrm{m}^{2}$. To produce these foams we used a neutral surfactant, Brij L-23, $\mathrm{C}_{12} \mathrm{E}_{23}$, or Polyoxyethylene(23)-lauryl ether, purchased from Aldrich.

\subsection{TOC measurements}


TOC, Total Organic Content, measurements (Shimadzu-VCSH) enable the amount of carbon in the solutions to be obtained, from which we can deduce the concentration of the free polymer in the solutions hence the amount of adsorbed molecules on the particles. We prepare PEI/silica mixtures containing $400 \mathrm{mg} / \mathrm{L}$ of silica particles and a PEI concentration ranging from 0 to $400 \mathrm{mg} / \mathrm{L}$. The $\mathrm{pH}$ is adjusted to 7 or 10 using a $1 \mathrm{M} \mathrm{NaOH}$ solution. The solutions are then sonicated for 5 minutes and then left to equilibrate for 24 hours under magnetic stirring. $2 \mathrm{~mL}$ of these particle-PEI mixtures were ultracentrifuged at $11000 \mathrm{rpm}$ for 24 hours (Beckman Coulter OptimaMAX Ultracentrifuge). The amount of Non Purgeable Organic Carbon (NPOC, given in $\mathrm{mg} \mathrm{L}^{-1}$ ) in the supernatant is measured by using the instrument. It is then converted into the molar concentration of the free PEI which was originally present in the supernatant referring to a calibration curve. The surface coverage of the particle by the polymer, $\Gamma$, is calculated from the following equation

$$
\Gamma=\frac{C_{\text {pei } 0}-C_{\text {peiTOC }}}{C_{\text {silica }} S_{\text {silica }}}
$$

where $C_{p e i 0}$ is the initial PEI concentration in the suspension, $C_{\text {реiтоc }}$ is the concentration of free PEI in the supernatant measured by the TOC experiment $C_{\text {silica }}$ is the silica concentration and $S_{\text {silica }}$ is the specific area of the silica particles. We note that the TOC measurements are performed for PEI/Silica suspensions without $\mathrm{C}_{12} \mathrm{E}_{23}$.

\subsection{Zeta potential measurements}

The zeta potential of the particles was calculated from the electrophoretic mobilities measured by using a Malvern Zetasizer Nano Series ZS90 instrument, using the Smoluchowski equation ${ }^{27}$. The samples used for this measurement were 250-fold diluted in Milli-Q water to reach a particle concentration of $0.01 \mathrm{wt} \%$ and avoid multiple scattering of light. At least 3 measurements were performed for each sample.

\subsection{Rheological measurements}

Rheological measurements were performed using an ARG2 rheometer from TA instruments equipped with a rough plane with $0.5 \mathrm{~mm}$ deep and $1 \mathrm{~mm}$ wide stripes to avoid slippage.

\subsection{Preparation of the gels and foams}


The gels were prepared at $\mathrm{pH} 7$ where the suspensions are in a fluid state and stirred for 24 hours. Then the $\mathrm{pH}$ is raised to 10.5 to reach the gel state and the neutral Brij surfactant is added to obtain the suspension to be foamed. We found using TOC measurements that the $\mathrm{C}_{12} \mathrm{E}_{23}$ adsorbs on the silica particles (see SI) therefore in the following the $\mathrm{C}_{12} \mathrm{E}_{23}$ concentration is fixed at equal to $3.3 \mathrm{~g} / \mathrm{L}$ to have a large excess of surfactant in excess in the suspension. As explained in the following the ratio of PEI to silica concentrations is fixed equal to 0.75 .

We produce foams with the silica/PEI gels using a planetary mixer (Kenwood Chef Premier KMC 560, $1000 \mathrm{~W}$ ) using an amount of initial liquid of $200 \mathrm{~mL}$ and at a velocity of $165 \mathrm{rpm}$. The variation of the air fraction of the foams with the mixing time is obtained by weighing known volumes of foamed suspensions.

To study the foam stability, the foams are poured into large glass syringes ( $25 \mathrm{~mm}$ diameter) covered with a cap to prevent evaporation. The volume of drained liquid is measured as a function of time by measuring the height of drained liquid using a camera. The size of the bubbles at the syringe walls is measured using a camera. We note that the size of wall bubbles may be larger than inside the foam, by a factor $\Pi^{1 / 2}$.

\section{Results}

\section{Characterization of the PEI/silica gels}

We use TOC and zeta potential measurements to characterize the adsorption of PEI on the silica particles. Figure 1 shows that for a suspension containing $400 \mathrm{mg} / \mathrm{L}$ of silica particles, the surface coverage of PEI on the particles increases to $0.6 \mathrm{mg} / \mathrm{m}^{2}$ and then reaches a plateau above $200 \mathrm{mg} / \mathrm{L}$ of PEI. The measured surface coverage is similar at $\mathrm{pH} 7$ and $\mathrm{pH} 10$ and does not depend on the salt concentration. The zeta potential, $\xi$, of the silica particles alone is negative at $\mathrm{pH} 7$ and it becomes positive above $3 \mathrm{mg} / \mathrm{L}$ of PEI as the positively charged PEI adsorbs on the particles. The zeta potential reaches a plateau above $20 \mathrm{mg} / \mathrm{L}$ of PEI. In the following we choose to work with a PEI/silica ratio of 0.75 corresponding to the plateau in surface excess, $\Gamma$, and zeta potential, $\xi$. For this PEI/silica ratio, the PEI coated silica particles become neutral at $\mathrm{pH}=10.5$ as the number of protonated groups on the PEI 
molecules decreases. At this $\mathrm{pH}$, in the absence of electrostatic repulsions, the PEI-coated particles strongly aggregate because of Van der Waals forces. We note that the point of zero charge is slightly different from what is reported in Ref 26 , which is probably due to the fact that the silica we use is purchased from a different supplier.

Consequently at $\mathrm{pH}=10.5$, the suspension turns into a strong gel, as shown in Figure 2, which presents G' and G' as a function of the stress amplitude. The yield stress is the critical stress above which G' drops and the deformation steeply increases ${ }^{28}$. The elastic modulus and the yield stress of the gel depend on the particle concentration. The elastic modulus ranges between $20 \mathrm{~Pa}$ and $10^{5} \mathrm{~Pa}$ as the silica concentration is raised from $12.8 \mathrm{wt} \%$ to $19.7 \mathrm{wt} \%$. The yield stress increases from $10 \mathrm{~Pa}$ to $2000 \mathrm{~Pa}$ when the silica concentration is increased from $12.8 \mathrm{wt} \%$ to $19.7 \mathrm{wt} \%$. We checked that the $\mathrm{C}_{12} \mathrm{E}_{23}$ does not have any influence on the rheological results.

\section{Foaming of the PEI/silica gels}

We produce foams with the silica/PEI gels using a kitchen mixer and we measure $\phi_{\text {air, }}$, the volume air fraction of the foams as a function of time. For the lowest silica concentration, $12,8 \mathrm{wt} \%$, $\phi_{\text {air }}$ rises to $80 \%$ in a few minutes and then reaches a plateau. For $15,5 \mathrm{wt} \%$ silica, $\phi_{\text {air }}$ reaches a maximum of $65 \%$ and then decreases with time. The $19.7 \mathrm{wt} \%$ silica suspension could not be foamed therefore $\phi_{\text {air }}$ remains below $8 \%$. Therefore our results show that increasing the particle concentration leads to lower $\phi_{\text {air. }}$. Similarly Lesov et al. ${ }^{17}$ found that increasing the particle concentration leads to less air entrapment in silica/surfactant mixtures. Indeed, they showed that increasing the particle concentration leads to an increase of the viscosity, which prevents air entrapment.

In an attempt to increase $\phi_{\text {air, }}$ we performed two step foaming experiments, similarly to the method we used for another gel system ${ }^{8}$. We first foam the PEI/silica suspension (containing the surfactant) at $\mathrm{pH} 7$ corresponding to the fluid state. Secondly the $\mathrm{NaOH}$ is added to the foam, which is continuously sheared in the mixer, to rise the $\mathrm{pH}$ up to 10.5 to reach the gel state. As shown in Figure 4, as the liquid suspension at $\mathrm{pH} 7$ is foamed, $\phi_{\text {air }}$ quickly rises to $80 \%$ for the $15.5 \mathrm{wt} \%$ silica suspension, which is higher than what is measured when the $15.5 \mathrm{wt} \%$ suspension is directly foamed in the gel state at pH10.5. However 
as $\mathrm{NaOH}$ is added, $\phi_{\text {air }}$ rapidly drops to $60 \%$ and remains constant afterwards. Therefore the gelation of the suspension does not enable to increase the air fraction in the foam and that in both processes the foams reach the same stationary state

\subsection{Stability of the PEI/silica foams}

After 25 minutes of foaming, $150 \mathrm{~mL}$ of foam were transferred into a glass syringe covered with a cap to record the stability of the foam to drainage and Oswald ripening. We find that the foam prepared with a $15.5 \mathrm{wt} \%$ suspension at $\mathrm{pH} 7$ is highly unstable and destabilizes in about an hour. After 30 minutes the size of the bubbles has increased from 0.1 to $2 \mathrm{~mm}$ and the total volume drains in about an hour.

The foams made with 12.8 and 15.5 wt $\%$ of silica particles and PEI at pH10.5 are much more stable to drainage and ripening. We do not detect any drained liquid for 1 hour. The size of the bubbles increases from $200 \mu \mathrm{m}$ to $2 \mathrm{~mm}$ for the $12.8 \mathrm{wt} \%$ silica suspension over an hour. For the $15.5 \mathrm{wt} \%$ suspension the size of the bubbles increases from $200 \mu \mathrm{m}$ to $400 \mu \mathrm{m}$ and then reaches a plateau after one hour.

For the 12.8 wt $\%$ suspension at $\mathrm{pH} 10.5$, the yield stress is $\sigma_{0}=10 \mathrm{~Pa}$. As shown by Lesov et al. and previously by Chaplain et al. ${ }^{29}$ when the yield stress $\sigma_{0}$ is higher than the buoyancy pressure $\Delta \mathrm{P}_{\text {buoy }}$, then the drainage flow is suppressed. This statement enables to define a critical yield stress which writes $\sigma_{0 c} \sim \Delta \mathrm{P}_{\text {buoy }} \sim \Delta \rho g R$, where $\Delta \rho$ is the mass density difference between bubbles and the surrounding suspension, $g$ is gravity acceleration and $\mathrm{R}$ is the bubble radius. Taking $\mathrm{R}=125 \mu \mathrm{m}$ we find $\sigma_{0 \mathrm{c}}=1,3 \mathrm{~Pa}$, which is below the yield stress of the $12.8 \mathrm{wt} \%$ suspension. Therefore the yield stress of the $12.8 \mathrm{wt} \%$ $\mathrm{PEI} /$ silica suspension is sufficiently high to prevent the drainage flow in agreement with our observations. However the foam bubbles ripen over time. According to Lesov et al. ${ }^{17}$, to arrest bubble Ostwald ripening, the yield stress has to overcome the difference in capillary pressure of the bubbles, which writes $\sigma_{0 c} \sim \Delta \mathrm{P}_{\text {cap }} \sim 2 \gamma / \mathrm{R}$, where $\gamma$ is the surface tension and $\mathrm{R}$ is the radius of the bubbles. At early times, $\mathrm{R}$ is of the order of $125 \mu \mathrm{m}$ and $\gamma=40 \mathrm{mN} / \mathrm{m}$ therefore $\Delta \mathrm{P}_{\text {cap }} \sim 640 \mathrm{~Pa}$, which is much higher than the yield stress of the $12.8 \mathrm{wt} \%$ suspension. Hence the yield stress is not sufficiently high to arrest Ostwald ripening. However in the case of the $15.5 \mathrm{wt} \%$ suspension, we find that the radius of the bubbles increases at early times and then it reaches a plateau of $400 \mu \mathrm{m}$ after an hour. Indeed the yield 
stress is $\sigma_{0}=200 \mathrm{~Pa}$, which is higher than $\Delta \mathrm{P}_{\text {buoy }}$ but lower than $\Delta \mathrm{P}_{\text {cap }}$ at early times. As time goes on, as the size of the bubbles increases, $\Delta \mathrm{P}_{\text {cap }}$ decreases. When $\mathrm{R}$ reaches $\mathrm{R}=2 \gamma / \sigma_{0} \sim 400 \mu \mathrm{m}$, the Ostwald ripening is theoretically arrested consistently with our measurements. We note that our results are not consistent with Kloek et al. numerical simulations, which predict that when the bulk shear elastic modulus of the aqueous phase is above $10^{4} \mathrm{~Pa}$ the Oswald ripening is completely arrested ${ }^{4}$. In our case, Oswald ripening is observed at early times for the $15.5 \mathrm{wt} \%$ suspension although the value of its shear elastic modulus is $10^{4} \mathrm{~Pa}$. In their simulations, Kloek et al. consider one single spherical bubble while in our case our bubbles are not spherical and surrounded by other bubbles.
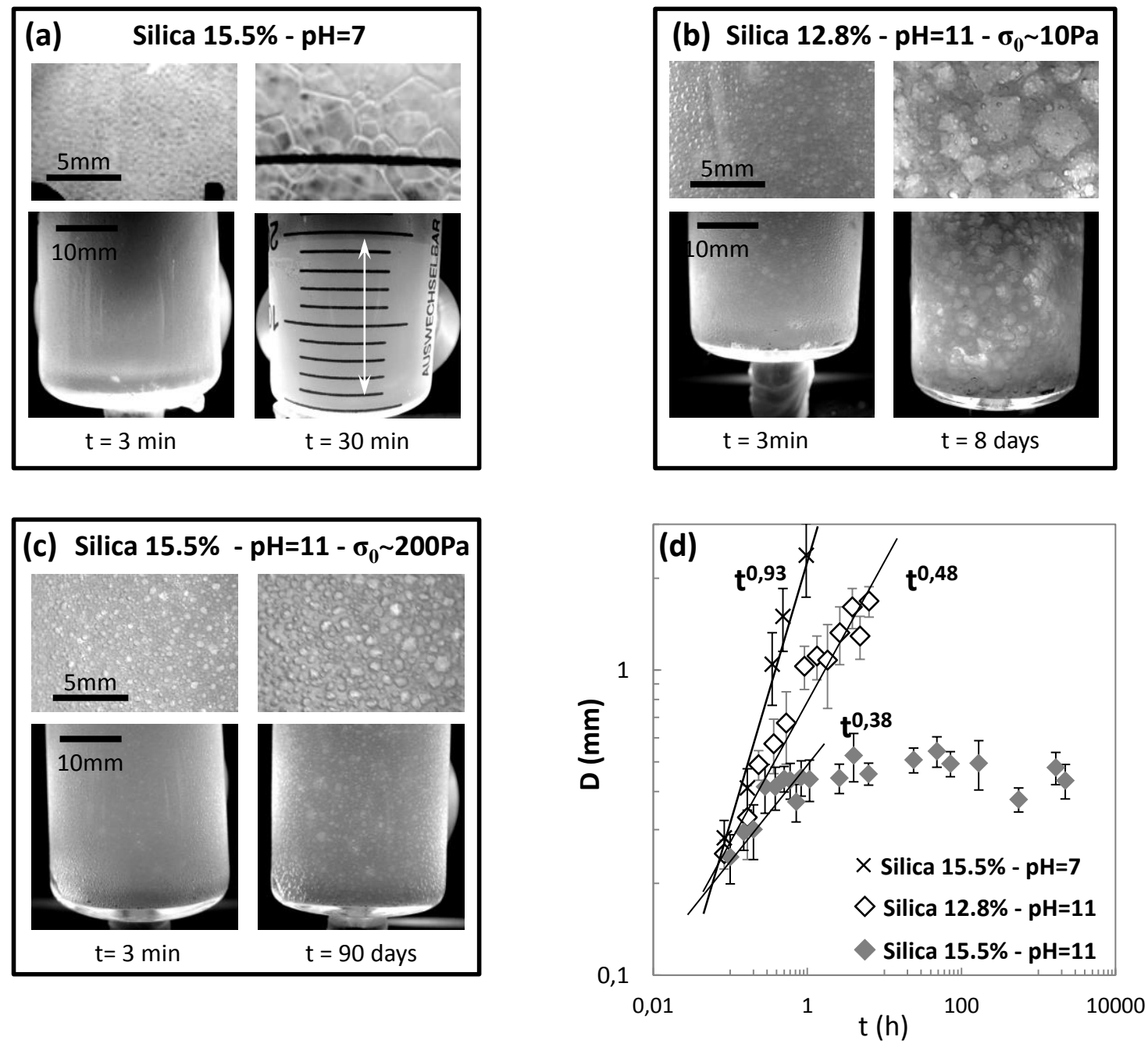

$<$ Inlinelmage6 $>$

Figure 6. Photographic image of the foams obtained with a) the PEI/silica suspension with 15.5 wt \% silica at $\mathrm{pH} 7, \mathrm{~b})$ the PEI/silica suspension with $12.8 \mathrm{wt} \%$ silica at $\mathrm{pH} 10.5, \mathrm{c})$ the PEI/silica 
suspension with 15.5 wt \% silica at pH10.5. d) mean diameter of the bubbles (measured at the syringe wall) as a function of time for the same suspensions as in a), b), c) $C_{12} E_{23}$ concentration $1.65 \mathrm{~g} / \mathrm{L}$

\section{Conclusion}

In this article we examined the foamability and foam stability of a series of PEI/silica gel suspensions with varying particle concentrations. These suspensions exhibit a finite yield stress whose value can be tuned by varying the particle concentration and the $\mathrm{pH}$ when all the suspensions are in the fluid state at $\mathrm{pH} 7$ - the air fraction is relatively high ( $80 \%$ for the $15,5 \mathrm{wt} \%$ suspension) however the foams are very unstable to drainage, when the suspensions are in the gel state - at $\mathrm{pH} 10.5-$, the obtained air fraction decreases for increasing suspension concentration, from $\phi_{\text {air }}=80 \%$ for the $12.8 \mathrm{wt} \%$ suspension to $\phi_{\text {air }}=8 \%$ for the $19.7 \mathrm{wt} \%$ suspension. The most concentrated suspension, i.e $19.7 \mathrm{wt} \%$ could not be foamed. In the gel phase - at pH10.5 - depending on the yield stress, the drainage and Oswlad ripening may be hindered. For a yield stress of about $10 \mathrm{~Pa}-$ at $12.8 \mathrm{wt} \%$ suspension - the buoyancy force is overcome and drainage is arrested. However it is too low to prevent Ostwald ripening. But a yield stress of $200 \mathrm{~Pa}$, is high enough to arrest drainage. At short times, Ostwald ripening is observed. At longer times as the size of the bubbles increases, the capillary pressure decreases and becomes lower than the yield stress. As a consequence the Ostwald ripening is arrested when the bubble radius reaches $400 \mu \mathrm{m}$ for a yield stress of $200 \mathrm{~Pa}$.

The results obtained in this study show that there is an optimal silica concentration which combines good foamability and large stability to drainage and Ostwald ripening. Moreover the yield stress appears to be a way to control the bubble radius. Moreover these PEI/silica suspensions are promising systems for the development of 3D printable porous materials even though the size of the bubbles, of the order of $400 \mu \mathrm{m}$, limits the spatial resolution that can be reached when printing the foamed gels. Finally the results obtained in this study confirm previously obtained results which show that drainage and coarsening can be arrested for sufficiently high yield stress.

\section{Acknowledgements}


The authors thank the ANRT and Saint-Gobain for financial support of this work as well as Global Station for Soft Matter, a project of Global Institution for Collaborative Research and Education at Hokkaido University and Mélanie Arangalage for performing preliminary measurements on PEI/silica foams.

\section{References}

1. Drenckhan, W. \& Saint-Jalmes, A. The science of foaming. Adv. Colloid Interface Sci. 222, 228-259 (2015).

2. Cohen-Addad, S., Höhler, R. \& Pitois, O. Flow in Foams and Flowing Foams. Annu. Rev. Fluid Mech. 45, 241-267 (2013).

3. Ramamurthy, K., Kunhanandan Nambiar, E. K. \& Indu Siva Ranjani, G. A classification of studies on properties of foam concrete. Cem. Concr. Compos. 31, 388-396 (2009).

4. Kloek, W., van Vliet, T. \& Meinders, M. Effect of Bulk and Interfacial Rheological Properties on Bubble Dissolution. J. Colloid Interface Sci. 237, 158-166 (2001).

5. Ducloué, L., Pitois, O., Goyon, J., Chateau, X. \& Ovarlez, G. Rheological behaviour of suspensions of bubbles in yield stress fluids. J. Non-Newton. Fluid Mech. 215, 31-39 (2015).

6. Salonen, A., Lhermerout, R., Rio, E., Langevin, D. \& Saint-Jalmes, A. Dual gas and oil dispersions in water: production and stability of foamulsion. Soft Matter 8, 699-706 (2012).

7. Testouri, A., Honorez, C., Barillec, A., Langevin, D. \& Drenckhan, W. Highly Structured Foams from Chitosan Gels. Macromolecules 43, 6166-6173 (2010).

8. Deleurence, R., Saison, T., Lequeux, F. \& Monteux, C. Time scales for drainage and imbibition in gellified foams: application to decontamination processes. Soft Matter 11, 7032-7037 (2015).

9. Andersen, T., Melvik, J. E., Gåserød, O., Alsberg, E. \& Christensen, B. E. lonically Gelled Alginate Foams: Physical Properties Controlled by Operational and Macromolecular Parameters. Biomacromolecules 13, 3703-3710 (2012).

10. Barbetta, A., Rizzitelli, G., Bedini, R., Pecci, R. \& Dentini, M. Porous gelatin hydrogels by gasin-liquid foam templating. Soft Matter 6, 1785 (2010).

11. Guillermic, R. M., Salonen, A., Emile, J. \& Saint-Jalmes, A. Surfactant foams doped with laponite: unusual behaviors induced by aging and confinement. Soft Matter 5, 4975 (2009).

12. Liu, Q., Zhang, S., Sun, D. \& Xu, J. Aqueous foams stabilized by hexylamine-modified Laponite particles. Colloids Surf. Physicochem. Eng. Asp. 338, 40-46 (2009).

13. Liu, Q., Zhang, S., Sun, D. \& Xu, J. Foams stabilized by Laponite nanoparticles and alkylammonium bromides with different alkyl chain lengths. Colloids Surf. Physicochem. Eng. Asp. 355, 151-157 (2010).

14. Sani, A. M. \& Mohanty, K. K. Incorporation of clay nano-particles in aqueous foams. Colloids Surf. Physicochem. Eng. Asp. 340, 174-181 (2009). 
15. Haffner, B., Khidas, Y. \& Pitois, O. The drainage of foamy granular suspensions. J. Colloid Interface Sci. 458, 200-208 (2015).

16. Rouyer, F., Haffner, B., Louvet, N., Khidas, Y. \& Pitois, O. Foam clogging. Soft Matter 10, 6990-6998 (2014).

17. Lesov, I., Tcholakova, S. \& Denkov, N. Factors controlling the formation and stability of foams used as precursors of porous materials. J. Colloid Interface Sci. 426, 9-21 (2014).

18. Lesov, I., Tcholakova, S. \& Denkov, N. Drying of particle-loaded foams for production of porous materials: mechanism and theoretical modeling. RSC Adv 4, 811-823 (2014).

19. Carn, F., Colin, A., Pitois, O., Vignes-Adler, M. \& Backov, R. Foam Drainage in the Presence of Nanoparticle-Surfactant Mixtures. Langmuir 25, 7847-7856 (2009).

20. Deleurence, R., Parneix, C. \& Monteux, C. Mixtures of latex particles and the surfactant of opposite charge used as interface stabilizers - influence of particle contact angle, zeta potential, flocculation and shear energy. Soft Matter 10, 7088-7095 (2014).

21. Gonzenbach, U. T., Studart, A. R., Tervoort, E. \& Gauckler, L. J. Ultrastable Particle-Stabilized Foams. Angew. Chem. Int. Ed. 45, 3526-3530 (2006).

22. Petit, P., Javierre, I., Jézéquel, P.-H. \& Biance, A.-L. Generation and stability of bubbles in a cement based slurry. Cem. Concr. Res. 60, 37-44 (2014).

23. Guignot, S., Faure, S., Vignes-Adler, M. \& Pitois, O. Liquid and particles retention in foamed suspensions. Chem. Eng. Sci. 65, 2579-2585 (2010).

24. Dickinson, E., Ettelaie, R., Kostakis, T. \& Murray, B. S. Factors Controlling the Formation and Stability of Air Bubbles Stabilized by Partially Hydrophobic Silica Nanoparticles. Langmuir 20, 85178525 (2004).

25. Surfactant foams doped with laponite: unusual behaviors induced by aging and confinement.

26. Smay, J. E., Gratson, G. M., Shepherd, R. F., Cesarano, J. \& Lewis, J. A. Directed Colloidal Assembly of 3D Periodic Structures. Adv. Mater. 14, 1279-1283 (2002).

27. Hunter, R. J. Zeta potential in colloid science: principles and applications. (Academic Press, 1981).

28. Mason, T. G., Bibette, J. \& Weitz, D. A. Yielding and Flow of Monodisperse Emulsions. J. Colloid Interface Sci. 179, 439-448 (1996).

29. CHAPLAIN, V (CHAPLAIN, V); MILLS, P (MILLS, P); GUIFFANT, G (GUIFFANT, G); CERASI, P (CERASI, P). MODEL FOR THE FLOW OF A YIELD FLUID THROUGH A POROUS-MEDIUM. Journal de Physique I/ 2145-2158 (1992). 

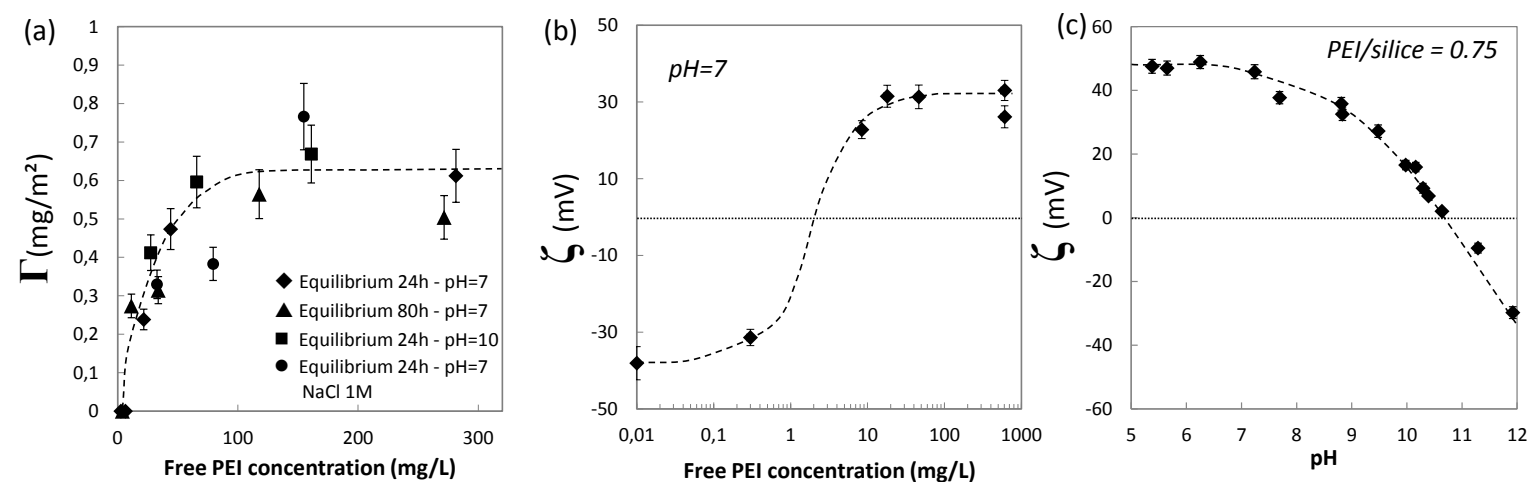

$<$ Inlinelmage2>

Figure 1. a) Surface coverage of PEI on the silica particles as a function of the free PEI concentration for suspensions without $\left.C_{12} E_{23} b\right)$ zeta potential of the silica particles as a function of the free PEI concentration at $\mathrm{pH} 7$ for $400 \mathrm{mg} / \mathrm{L}$ of silica particles, C) zeta potential as a function of $\mathrm{pH}$ for $\mathrm{PEI} /$ silica ratio of 0.75 , ie silica concentration $400 \mathrm{mg} / \mathrm{L}$ and PEI concentration of $300 \mathrm{mg} / \mathrm{L}$. Dashed lines are guides for the eye to see the trends.

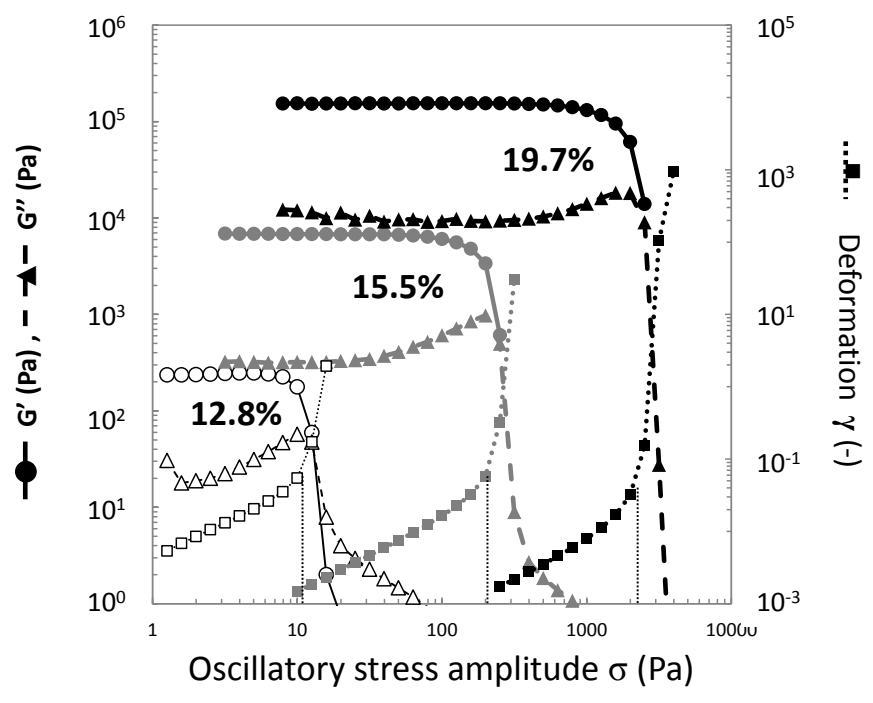

$<$ Inlinelmage3>

Figure 2. Storage modulus, $G$, loss modulus, $G$ "' and deformation amplitude as a function of oscillatory shear amplitude, for PEI/Silica suspensions at pH 10.5, with various Silica concentration, keeping a PEI/silica ratio of 0.75 . Vertical lines stand for the yield stress, determined as the stress for which $G$ ' collapses and the slope of the deformation/stress curve changes, according to Reference 28. 

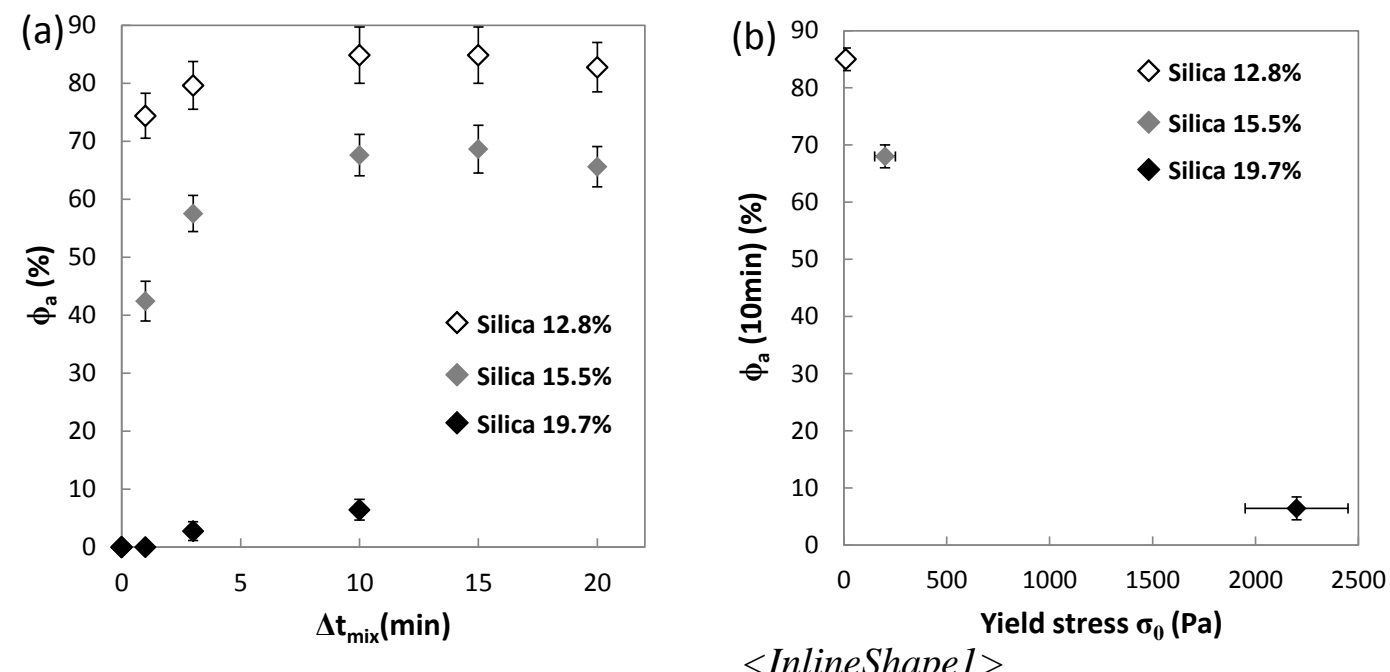

Figure 3. (a) Air fraction incorporated into the foams as a function of the shearing time for three particle concentrations, $12.8 w t \%, 15.5$ wt \%and $19.7 w t \%$. The $C_{12} E_{23}$ concentration is $1.65 \mathrm{~g} / \mathrm{L}$. and $\mathrm{pH}=10.5(\mathrm{~b})$ Value of the air fraction after 10 minutes mixing as a function of the silica content
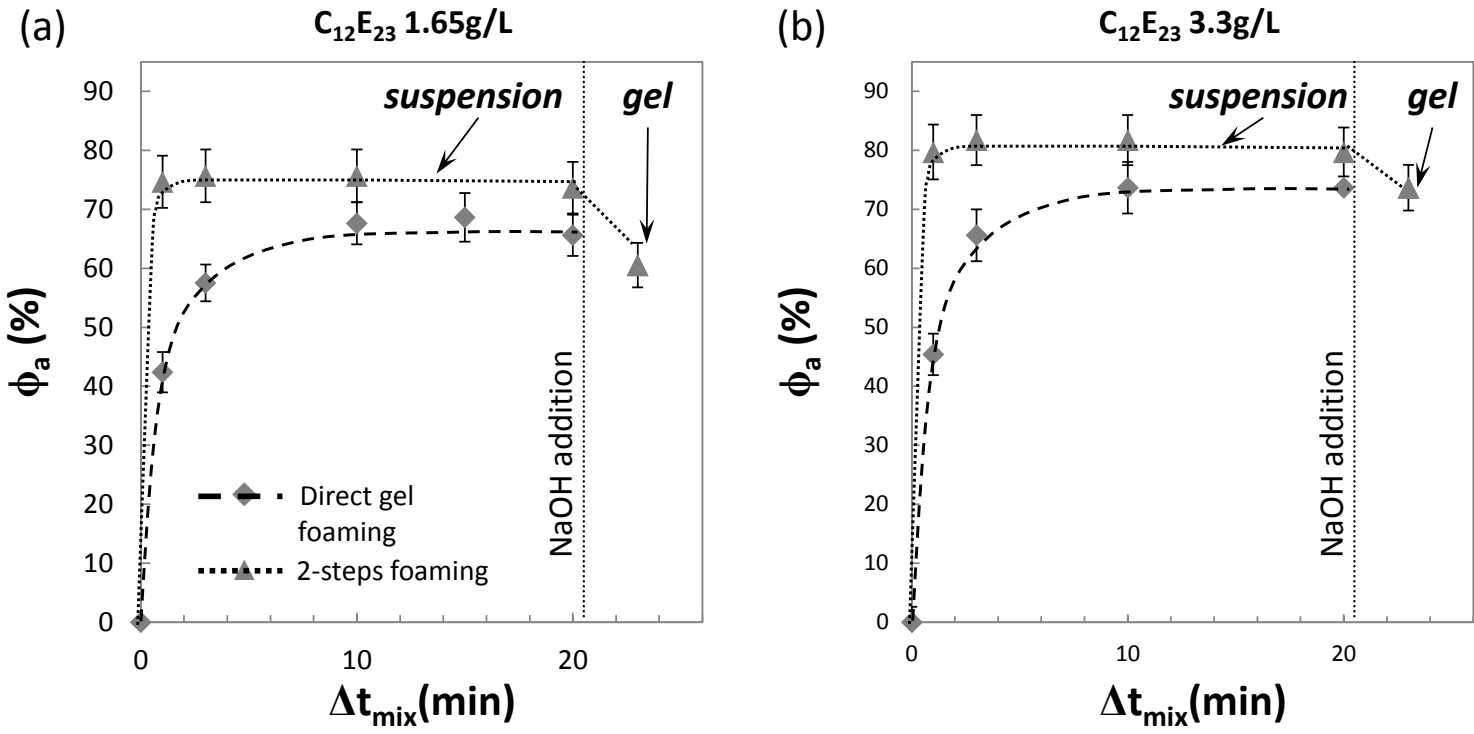

$<$ Inlinelmage4>

Figure 4. Air fraction as a function of time during the two-step foaming process for PEI/silica suspension containing $15.5 \mathrm{wt} \%$ silica, $11.6 \mathrm{wt} \%$ PEI and $1.65 \mathrm{~g} / \mathrm{L}$ of $\mathrm{C}_{12} E_{23}$ in a), and $3.3 \mathrm{~g} / \mathrm{L}$ of $C_{12} E_{23}$ in $b$. In $a$ ) and $b$ ), the suspension is foamed at pH7 as the suspension is fluid and then the $\mathrm{NaOH}$ is added to obtain a strong gel. The foaming of the suspension in the fluid state is higher 
than when the suspension is foamed at pH10.5 directly, however as $\mathrm{NaOH}$ is added, the air volume fraction decreases from $75 \%$ to $60 \%$ in a), and from $80 \%$ to $70 \%$ in b).

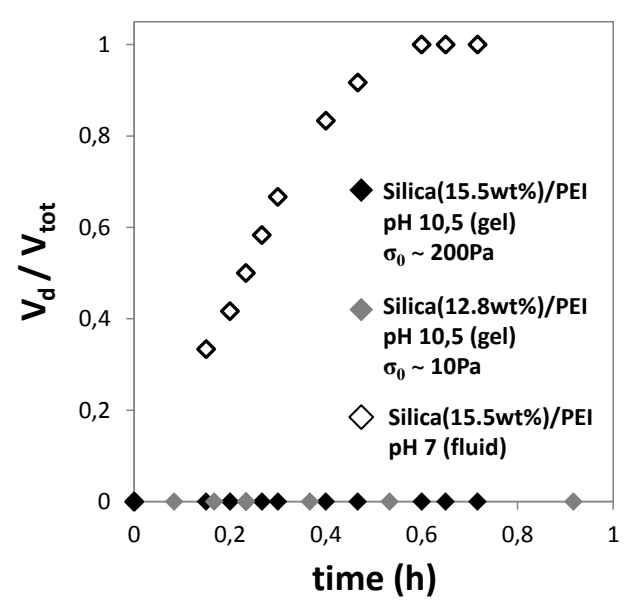

$<$ Inlinelmage5>

Figure 5. Drainage flow of the PEI/silica foams with varying silica concentrations. $V_{d} / V_{\text {tot }}$ as a function of time. $V_{d}$ is the volume of drained liquid and $V_{\text {tot }}$ is the total volume of drained liquid. $C_{12} E_{23}$ concentration is $1.65 \mathrm{~g} / \mathrm{L}$ 